Int. J. Odontostomat., 9(1):159-164, 2015.

\title{
Características de los Canales Radiculares de Molares Temporales
}

\author{
Characteristics of Roots Canals of Deciduous Teeth
}

Esther Vaillard Jiménez*; Enrique Huitzil Muñoz** \& Loida Castillo Domínguez**

VAILLARD, J. E.; HUITZIL, M. E. \& CASTILLO, D. L. Características de los canales radiculares de molares temporales. Int. J. Odontostomat., 9(1):159-164, 2015.

RESUMEN: El objetivo de esta investigacion fue identificar en los canales radiculares de la dentición temporal las variantes anatómicas de la cavidad dentaria pulpar. Se realizó un estudio clínico, descriptivo, transversal ex vivo, que analizó una muestra de 60 dientes temporales pertenecientes a niños mexicanos aparentemente sanos, cuya extracción se indicó por motivos terapéuticos. Los dientes fueron almacenados en agua destilada y posteriormente diafanizados. La identificación de canales radiculares se realizó por un solo observador durante dos ocasiones (k=0,7947). Los dientes unirradiculares presentaron $50 \%$ de canales simples y laterales, $25 \%$ secundarios y $25 \%$ deltas. Los molares maxilares presentaron un $8 \%$ de canales simples en cada raíz, mientras que los molares mandibulares un $29 \%$. Canales secundarios, paralelos y recurrentes se observaron en el $33 \%$ de los molares. El $100 \%$ de las raíces distales de molares mandibulares presentaron canales reticulares. Las fusiones radiculares se presentan en el $88 \%$ de las molares mandibulares. La anatomía de los canales radiculares de la dentición temporal es altamente compleja.

PALABRAS CLAVE: canales radiculares, dentición temporal, diafanización dental.

\section{INTRODUCCIÓN}

Ante la mayor demanda de tratamientos de canales radiculares y accesibilidad al tratamiento endodóntico, resulta necesario examinar las características del sistema de canales para identificarlos de manera efectiva, conocer sus particularidades y establecer protocolos de diagnóstico y tratamiento que aseguren el éxito y rehabilitación de la función masticatoria. Por otro lado, existen variaciones anatómicas importantes en la forma de las raíces dentarias y sus canales, que resultan relevantes registrar en cuanto a su localización y forma longitudinal y transversal para garantizar el éxito de la terapia endodóntica.

Se reconocen como componentes del sistema pulpar a la cavidad pulpar, como el espacio central de un diente, ubicada dentro de la estructura coronaria y radicular del diente. Los canales radiculares corresponden a las estructuras de la raíz dental que inclu- yen a los foramenes de entrada de los canales, canales propiamente dichos que pueden ser reconocidos como principales, y que se acompañan en la mayoría de los casos de canales accesorios que pueden terminar o no en un foramen apical.

La forma de los canales radiculares varía de acuerdo con la forma de la raíz dentaria; si ésta es curva, el canal principal le seguirá y por tanto resultará ser también curvo. Sin embargo, las raíces dentarias contienen más de un canal y algunos de ellos se denominan accesorios o laterales, que resultan ser ramas del canal radicular principal y que por lo general se presentan en el tercio apical o en área de la bifurcación de un diente multirradicular. Tales anomalías se presentan con cierta frecuencia en los dientes incisivos laterales maxilares, premolares mandibulares y molares maxilares, y tienden a presentarse bilateralmente (Walton \& Vertucci, 1997).

\footnotetext{
* Maestra en Estomatología Pediátrica. Docente de la Facultad de Estomatología, Benemérita Universidad Autónoma de Puebla, Puebla, México. "* Maestro en Educación Superior Docente de la Facultad de Estomatología, Benemérita Universidad Autónoma de Puebla, Puebla, México.

"*t*Alumna de segundo año de la maestría en Ciencias Estomatológicas en Pediátrica, Estomatología, Benemérita Universidad Autónoma de Puebla, Puebla, México.
} 
La remoción completa del tejido pulpar alterado, la limpieza de las paredes de la cavidad dentaria, su acondicionamiento y desinfección para recibir los materiales de obturación está muchas veces condicionada por la anatomía interna de los canales radiculares de la raíz dentaria.

En la literatura se refieren variaciones anatómicas de los canales radiculares principales de los dientes permanentes, acompañados muchas veces de canales radiculares accesorios y secundarios; condición que se presenta en los dientes incisivos centrales y caninos maxilares. En los dientes incisivos laterales es muy reconocida la pronunciada curvatura en el tercio apical radicular. También se han encontrado canales radiculares recurrentes y laterales. Los dientes incisivos mandibulares se caracterizan por la relativa falta de curvaturas en su raíz; sin embargo suelen identificarse dos canales radiculares, que pueden recorrer la raíz por separado, reunirse en un punto y tener solo una salida o volver a separarse para salir. Se han identificado entre ellos canales radiculares inter recurrentes. Su zona apical radicular puede tener una curvatura y canales radiculares accesorios. Los dientes caninos mandibulares suelen tener dos raíces fusionadas o separadas en el tercio apical, por lo que pueden tener dos forámenes apicales. Las dientes molares maxilares muestran tres raíces; la más larga es la palatina. En el diente primer molar maxilar la raíz distal es por lo general recta y cónica, y solo contiene un canal; sin embargo, la raíz mesial puede tener dos forámenes apicales porque están unidas la bucal y la palatina, por lo que pueden presentar una serie de combinaciones en el recorrido de sus canales semejantes a las de los dientes premolares.

En el segundo molar maxilar se aprecian frecuentemente fusiones radiculares y curvaturas poco marcadas. Generalmente presentan dos canales radiculares, uno palatino y el bucal. Cuando las raíces se presentan separadas, cada una suele contener solo un canal radicular, y puede presentar uno recurrente.

En los primeros molares mandibulares, suelen presentarse solo dos raíces, cada una con su canal radicular; sin embargo, muchas veces se encuentran dos canales radiculares en la raíz mesial y dos en la distal. Los canales radiculares mesiales suelen ser muy curvos mesio-bucalmente y tener una salida (Cohen \& Burns, 2002).

En cuanto a la dirección se refiere, los canales radiculares siguen el mismo eje de la raíz y la acom- pañan en todas sus canales. Por lo general, el $97 \%$ de los canales radiculares son curvos y pueden tener ramificaciones.

De acuerdo con lo referido por Kuttler (1986), las ramificaciones fueron clasificadas por Okumura con una nomenclatura relativamente sencilla que fue complementada por Pucci \& Reig (1945) al agregarle el canal cavo- interradicular.

Las ramificaciones del canal radicular principal pueden ser: i) Colateral o paralelo al canal principal; ii) Lateral que sale del principal hacia una pared del canal y tiene salida; iii) Interrecurrente, es el canalículo que une al canal radicular principal con el colateral; iv) Recurrente, es el canal radicular que sale, forma un asa y regresa al canal radicular principal del que salió; v) Secundario, es el que sale del canal radicular principal o del colateral, que tiene salida en el tercio apical, pero que no forma parte del delta apical; vi) Accesorio, es aquel canal radicular que sale de un canal radicular secundario y tiene salida en el tercio apical; vii) Cavo inter-radicular, es que sale directamente de la cavidad pulpar y tiene salida en el piso de la bifurcación, y viii) Delta complementarios, salen del canal radicular principal antes de la salida por el foramen apical (Kutler).

Los canales radiculares reticulares son pequeños canalículos entrelazados en forma de red que forman ramificaciones que pueden recorrer toda la raíz (Lasala, 1979).

El número de canales es otro punto importante a tratar en la descripción de la anatomía radicular. Vertucci (2005) hace una clasificación respecto al número de canales radiculares como:

1. Un canal radicular simple.

2. Dos canales radiculares iniciales que tienen una sola terminación y salida apical

3. Un canal radicular que se separa y se vuelve a unir para tener una salida apical.

4. Dos canales radiculares separados en una sola raíz. 5. Un canal radicualr que se divide en el tercio medio radicular y tiene dos salidas apicales.

6 . Salen de la cavidad pulpar de la corona dos canales radiculares, se unen en un segmento y se separan para tener dos salidas apicales.

7. Un canal radicular que se separa en dos, se une, se separa en un segmento y tiene una salida apical.

El número de raíces fusionadas es importante 
para identificar el número de canales radiculares que pueda tener cada raíz en fusión.

Los canales radiculares dentarios accesorios, se definen como comunicaciones entre el tejido pulpar y el ligamento periodontal que no involucran a la terminación apical. Son el resultado de fallas en la formación de la vaina epitelial radicular durante la fase embrionaria de la odontogenesis. Estos canalículos permiten la difusión de productos de descomposición tisular pulpar o de infecciones bacterianas en los tejidos periodontales (Kumar, 2009).

La información que la literatura reporta se refiere a la dentición permanente. En la actualidad se considera a la dentición temporal como estructuras semejantes a las de dientes permanentes; sin embargo, existen diferencias importantes en la forma y distribución de los canales radiculares dentarios. Los dientes incisivos centrales y laterales maxilares temporales tienen un canal radicular que sigue la forma de la raíz que en el tercio apical muestra una curvatura; el diente incisivo central hacia palatino y el incisivo lateral hacia distal, y son para esquivar al germen dental del diente incisivo permanente; se les reconoce como curva de compensación. Los dientes incisivos centrales y laterales mandibulares también tienen una sola raíz que suele ser recta y angosta, plana y redonda. En algunos dientes incisivos se aparece un ligero surco en medio de toda la raíz. Los dientes caninos temporales también son uni-radiculares y muestran curvaturas en el segmento apical. Algunos muestran un surco mediano que recorre toda la raíz. Los molares temporales maxilares tienen tres raíces largas, delgadas y divergentes; cada una tiene un canal radicular principal. En algunos casos las raíces palatinas están unidas por un puente de cemento con alguna raíz bucal. Las molares temporales mandibulares suelen tener solo dos raíces en las que se observan fusiones entre las raíces mesiales o entre las distales y en ocasiones en ambos lados, por lo que suelen tener hasta cuatro canales radiculares (Vaillard-Jiménez, 2012).

Sin embargo, en la dentición temporal es muy limitado el trabajo mecánico de las limas debido a la delgadez y curvaturas de las raíces y muchas veces el fracaso de las pulpectomías se atribuye a una mala técnica de instrumentación porque se desconocen las características de los canales y sus variantes, por lo que se plantea como objetivo en este trabajo identificar en los canales radiculares de la dentición temporal las variantes anatómicas de la cavidad pulpar.

\section{MATERIAL Y MÉTODO}

Previa autorización de la comisión de investigación de la Facultad de Estomatología de la Benemérita Universidad Autónoma de Puebla (BUAP), se realizó en las instalaciones de la Facultad de Estomatología durante el año 2012 un estudio clínico, descriptivo, transversal ex vivo en el que se analizó una muestra de 60 dientes temporales pertenecientes a niños mexicanos aparentemente sanos, en los que se indicó su extracción por motivos terapéuticos. Los dientes se almacenaron en agua destilada a temperatura ambiente y se diafanizaron de acuerdo a la técnica descrita por Sánchez Tecopala et al. (2011) que se modificó para aplicarse en dientes temporales.

En la fase de preparación y limpieza los dientes solo se lavaron con hipoclorito de sodio al $5 \%$ y se enjuagaron en agua corriente durante 5 minutos, después se abrieron las cavidades pulpares con fresa de carburo 329 y se identificaron las entradas de los canales radiculares con una lima 15 . Sin penetrar en ellos, se lavaron con hipoclorito de sodio al $5 \%$ y se enjuagaron con agua destilada para después inyectarles tinta china para su tinción. Una vez llenos los canales con tinta china se taparon y sellaron las coronas dentarias con cera.

La fase de descalcificación se logró con ácido nítrico al $3 \%$ y se sumergieron a los dientes incisivos y caninos solo durante una hora con constantes revisiones para evitar la desaparición del segmento apical. Las molares se descalcificaron durante 2 horas con $30 \mathrm{mi}-$ nutos, con las mismas precauciones para evitar que segmentos radiculares muy delgados desaparecieran.

El lavado posterior a la exposición al ácido nítrico se hizo sumergiéndolos en $500 \mathrm{~mL}$ de agua destilada, durante 15 minutos. Se secaron con papel secante antes de someterlos a la fase de deshidratación en diferentes concentraciones de alcohol desnaturalizado que iniciaron al $80 \%$, seguida por la concentración de alcohol al $90 \%$ y finalizar en alcohol sin diluir en su presentación comercial de $96^{\circ}$. En cada concentración permanecieron sumergidos durante solo una hora.

La fase de transparentación se logró al sumergir los órganos dentarios en salicilato de metilo durante 24 horas. Para conservarlos transparentes se conservaron en Xilitol.

La identificación de canales se realizó por un solo observador durante dos ocasione $(k=0,7947)$. 
RESULTADOS. Se logró la diafanización de todos los dientes tratados. Los canales radiculares se clasificaron de acuerdo a las taxonomías de Okamura y Vertucci, y analizaron de acuerdo a su condición uni o multirradicular y de acuerdo al arco maxilar o mandibular.
Las frecuencias en que se presentan los diferentes canales radiculares dentarios aparecen las Tablas I, II y III de acuerdo a las características uni o multirradiculares de cada diente. Se calculó el intervalo de confianza al $95 \%$ para cada proporción referida de acuerdo al tipo de canal radicular.

Tabla I. Distribución de frecuencias de la tipología de canales radiculares para dientes unirradiculares de acuerdo a la clasificación de Okamura y de Vertucci

\begin{tabular}{|c|c|c|c|c|c|c|}
\hline & ICS & ILS & IC I & ILI & $\mathrm{CS}$ & C I \\
\hline \multicolumn{7}{|c|}{ Clasificación de Okamura } \\
\hline Simple & $50 \pm 27,2 \%$ & $67 \pm 25,6 \%$ & $50 \pm 27,2 \%$ & $50 \pm 27,2 \%$ & $100 \%$ & $100 \%$ \\
\hline Lateral & --- & $33 \pm 25,6 \%$ & --- & $50 \pm 27,2 \%$ & --- & --- \\
\hline Secundario & $25 \pm 23,5 \%$ & --- & $50 \pm 27,2 \%$ & --- & --- & --- \\
\hline Delta & $25 \pm 23,5 \%$ & --- & --- & --- & --- & --- \\
\hline \multicolumn{7}{|c|}{ Clasificación de Vertucci } \\
\hline V1 & $50 \pm 27,2 \%$ & $100 \%$ & $50 \pm 27,2 \%$ & $100 \%$ & $100 \%$ & $100 \%$ \\
\hline $\mathrm{V} 3$ & $25 \pm 23,5 \%$ & --- & --- & --- & --- & --- \\
\hline V5 & $25 \pm 23,5 \%$ & --- & $50 \pm 27,2 \%$ & --- & --- & --- \\
\hline
\end{tabular}

Se reportan los valores de los intervalos de confianza al 95\% para las proporciones.

Tabla II. Tipos de fusión radicular y número de canales radiculares dentarios.

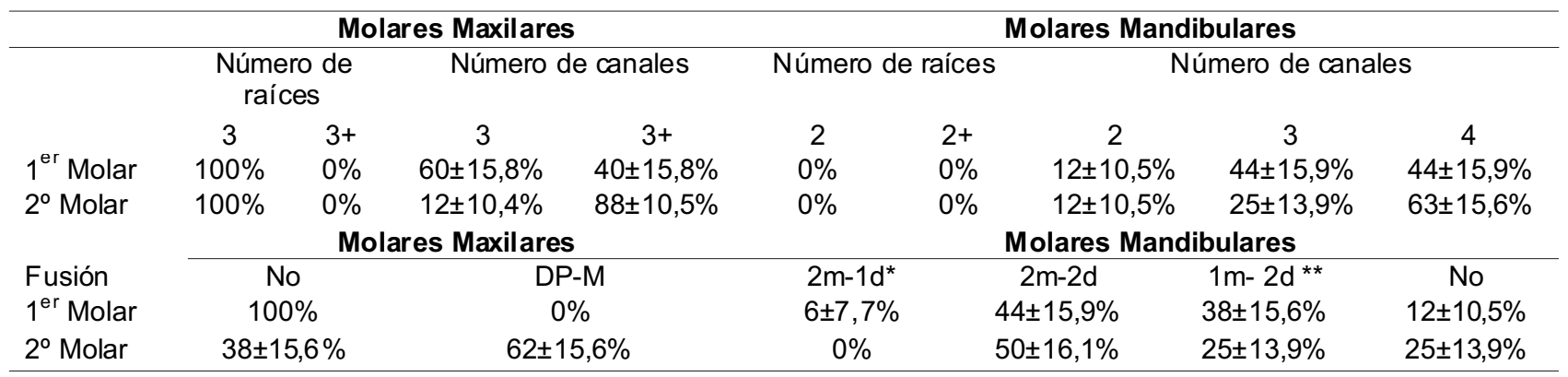

*La combinación de raíces fusionadas de las molares mandibulares se presentan como dos raíces mesiales fusionadas y la distal separada. ${ }^{* *}$ Existen casos en que la raíz separada es la mesial y las distales están fusionadas.

Tabla III. Distribución de la tipología de canales radiculares para dientes multirradiculares de acuerdo a la clasificaciones de Okamura y Vertucci.

\begin{tabular}{|c|c|c|c|c|c|c|c|c|c|c|}
\hline \multirow{4}{*}{$\begin{array}{l}\text { Tipo } \\
\text { Canal }\end{array}$} & \multicolumn{6}{|c|}{ Maxilares } & \multicolumn{4}{|c|}{ Mandibulares } \\
\hline & \multicolumn{8}{|c|}{ Clasificación de Okamura } & & \\
\hline & \multicolumn{3}{|c|}{$1^{\mathrm{er}}$ Molar } & \multicolumn{3}{|c|}{$2^{\circ}$ Molar } & \multicolumn{2}{|c|}{$1^{\mathrm{er}}$ Molar } & \multicolumn{2}{|r|}{$2^{\circ}$ Molar } \\
\hline & M & $\mathrm{D}$ & $\mathrm{P}$ & M & $\mathrm{D}$ & $\mathrm{P}$ & M & $\mathrm{D}$ & M & $\mathrm{D}$ \\
\hline Reticular & --- & --- & --- & --- & --- & -- & $25 \pm 13,9 \%$ & $100 \%$ & --- & $100 \%$ \\
\hline Simple & $8 \%$ & $8 \%$ & $8 \%$ & $\begin{array}{r}8 \% \\
\pm 8,74\end{array}$ & $8 \%$ & $8 \%$ & \multicolumn{2}{|l|}{ $\pm 14,6$} & \multicolumn{2}{|c|}{ $\pm 13,12$} \\
\hline Paralelo & $33 \%$ & --- & $33 \%$ & $33 \%$ & $67 \pm 15,15 \%$ & -- & --- & --- & --- & --- \\
\hline Secundario & --- & $33 \%$ & --- & $33 \%$ & --- & - & --- & $33 \%$ & --- & --- \\
\hline \multirow[t]{2}{*}{ Recurrente } & --- & --- & --- & $33 \%$ & --- & -- & --- & $33 \%$ & $33 \%$ & --- \\
\hline & \multicolumn{3}{|c|}{ $\pm 15,15$} & & --- & -- & --- & \multicolumn{2}{|c|}{ $\pm 15,15$} & --- \\
\hline Cavo-interradicular & \multicolumn{3}{|c|}{--- } & & --- & -- & $6,3 \pm 11,91 \%$ & \multicolumn{2}{|c|}{---} & $12,3 \pm 22,9 \%$ \\
\hline \multicolumn{11}{|c|}{ Clasificación de Vertucci } \\
\hline V1 & \multicolumn{3}{|c|}{$29 \pm 14,6 \%$} & \multicolumn{3}{|c|}{$36 \pm 15,5 \%$} & \multicolumn{2}{|c|}{$14 \pm 11,2 \%$} & \multicolumn{2}{|r|}{$21 \pm 13,12 \%$} \\
\hline V2 & \multicolumn{3}{|c|}{---} & \multirow{2}{*}{\multicolumn{3}{|c|}{$\begin{array}{l}7 \pm 8,22 \% \\
9 \pm 9,22 \%\end{array}$}} & \multicolumn{2}{|l|}{--- } & \multicolumn{2}{|r|}{---} \\
\hline V4 & \multicolumn{3}{|c|}{---} & & & & \multicolumn{2}{|c|}{$67 \pm 15,15 \%$} & \multicolumn{2}{|r|}{$24 \pm 13,8 \%$} \\
\hline V5 & \multicolumn{3}{|c|}{$8 \pm 8,74 \%$} & \multicolumn{3}{|c|}{---} & \multicolumn{2}{|l|}{--- } & \multicolumn{2}{|r|}{--- } \\
\hline
\end{tabular}




\section{DISCUSIÓN}

En la literatura no existen reportes sobre estudios de las características de los canales radiculares de la dentición temporal, tampoco los hay sobre técnicas específicas de diafanización para ellos (Chegüe-Vargas et al., 2007). La aplicación de los criterios taxonómicos para la dentición permanente se probó y resultó útil en la dentición temporal.

En algunos casos la diafanización de algunos dientes temporales no resulta ser didáctica porque en la fase de tinción, no se aprecian los detalles porque se trata de

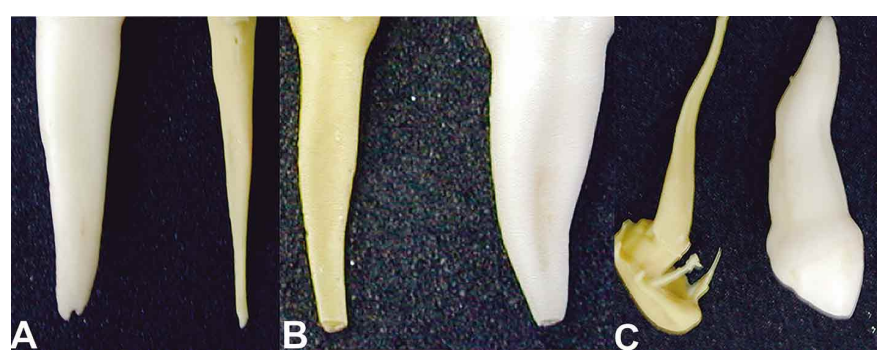

Fig. 1. Impresiones tomadas a canales radiculares dentarios unirradiculares. A. Canal radicular de un diente incisivo central mandibular; B. Canal radicular de un diente incisivo central maxilar con el surco central que recorre toda la raíz y reproduce la curvatura compensatoria; C. Canal radicular de un diente canino maxilar.

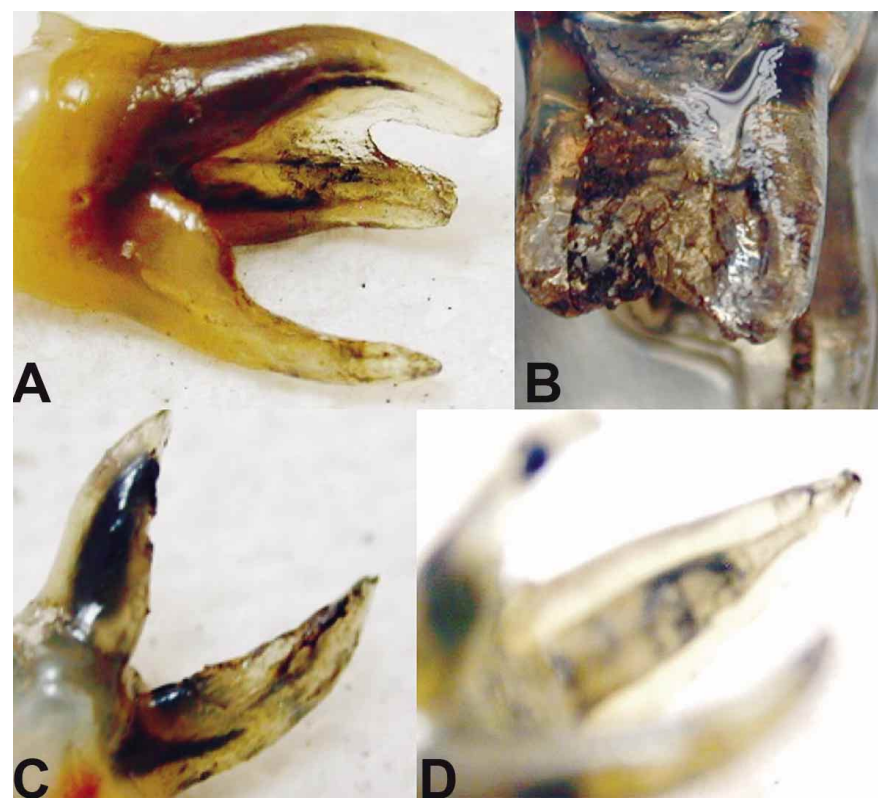

Fig. 2. Molares temporales diafanizados. A. Canal radicular dentario colateral; B. Canal radicular dentario en la raíz distal de un segundo molar temporal mandibular; C. Canal radicular dentario recurrente; D. Canal radicular dentario lateral e inter-recurrente. espacios muy grandes. La posibilidad de encontrar un canal radicular dentario con un surco central no es viable con la diafanización, para lo que es recomendable la impresión con silicón ligero (Fig. 1), donde también es posible demostrar las curvaturas y el adelgazamiento proporcional en la zona apical (Fig. 1).

Las clasificaciones de Okamura y Vertucci coinciden solo en los canales radiculares simples, pero es causa de confusión considerar a un canal radicular recurrente como un canal que se separa y se reúne al canal radicular principal (Fig. 2). En el caso de canales radiculares colaterales no se identifican sus entradas desde la cavidad pulpar coronal ni durante su trayectoria y no son susceptibles de ser instrumentados (Fig. 2).

Los canales radiculares reticulares se presentaron en la totalidad de las muestras de segundos molares mandibulares analizados, algunas de ellas mostraron patrones de reabsorción externa asociadas con infecciones óseas (Fig. 2B).

A diferencia de lo reportado por Kumar quien con la ayuda del microscopio electrónico de barrido cuantificó las dimensiones de este tipo de canales radiculares accesorios que conectan al piso de la cavidad pulpar coronaria con la bifurcación, también conocidos como canalículos cavo interradiculares; los encontró con una frecuencia del $53,33 \%$ en una muestra de 60 molares temporales. Con la técnica de diafanización solo se identificaron en los molares mandibulares; el $6 \%$ de los primeros molares y $12 \%$ de los segundos molares. Las combinaciones de más de un canal radicular accesorio resulta riesgosa para las pulpectomías (Fig. 2D).

Los deltas en el tercio apical radicular de los dientes incisivos maxilares se componen de canales diminutos difíciles de identificar (Fig. 3A) y en los que tal vez tampoco sea efectiva la acción de los irrigantes para canales infectados, razón por la cual los ejemplares analizados mantuvieron su longitud radicular, ya que las historias clínicas de los pacientes a los que pertenecieron las muestras, mostraron infección ósea. La terminación bífida del tercio apical radicualar no aparece como parte de ambas clasificaciones de canales (Fig. 3B-C). 


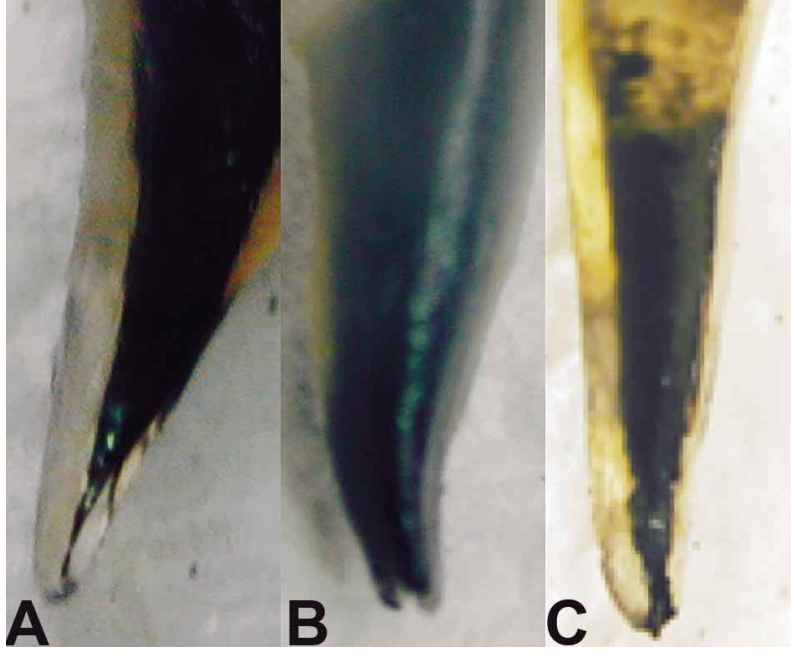

Fig. 3. A. Canales radiculares dentarios delta complementarios de Okamura; B y C. Canal radicular dentario dos salidas apicales.

VAILLARD, J. E.; HUITZIL, M. E. \& CASTILLO, D. L. Characteristics of roots canals of deciduous teeth. Int. J. Odontostomat., 9(1):159-164, 2015.

ABSTRACT: The aim of this study was to identify in roots canals of deciduous teeth anatomical variants in pulpar cavity. In a clinical, descriptive, cross sectional ex vivo study was analyzed a sample of 60 deciduous teeth of healthy Mexican children. Each tooth was conserved in distillated water and were transparency with a technic modified for deciduous teeth. Identifications of root canals was made twice by a lonely observant $(k=0.7947)$. Simple root teeth show $50 \%$ simple and lateral root canal, $25 \%$ secondary, $25 \%$ delta. Upper molars show $8 \%$ simple canal in each root. Lower molars $29 \%$. Secondary root canals, parallel and recurrent were identified in $33 \%$ of cases. $100 \%$ of distal roots of lower molars have reticular canals. Root fusions are presents in $88 \%$ of lower molars. In conclusion, root canals anatomy of deciduous teeth is high complex. tal transparency.

KEY WORDS: root canals, deciduous teeth, den-

\section{REFERENCIAS BIBLIOGRÁFICAS}

Chegüe-Vargas, N.; Cervantes-Amador, F.; Moreno-Castillo, E. N.; Espinosa-Reyes, I. \& Bautista-Pastrana, M. C. Técnica de diafanización en dientes humanos extraídos como material didactico para el conocimiento del sistema de conductos radiculares. Med. Oral, 9(3):78-80, 2007.
Cohen, S. \& Burns, R. C. Pathways of the pulp. 8a ed. St. Louis, Mosby, 2002.

Kumar, V. D. A scanning electron microscope study of prevalence of accessory canals on the pulpal floor of deciduous molars. J. Indian Soc. Pedod. Prev. Dent., 27(2):85-9, 2009.

Kuttler, Y. Fundamentos de Endo Metaendodoncia Práctica. $3^{\mathrm{a}}$ ed. México D. F., Fco. Mendez Ed., 1986.

Lasala, A. Endodoncia. $3^{a}$ ed. Barcelona, Salvat Editores, 1979.

Pucci, F. M. \& Reig, R. Conductos radiculares. Montevideo, Barreiro y Ramos, 1945.

Sanchez Tecopala, E.; Furuya Meguro, A. T. \& Llamoss Hernández, E. Metodología para la diafanización de dientes extraídos con cubierta de resina cristal y esmalte intacto. Endod. Actual, 6(16):6-12, 2011.

Vaillard-Jiménez, E. Anatomía de la dentición temporal. Puebla, ACD, 2012.

Vertucci, F. J. Root canal morphology and its relationship to endodontic procedures. Endod. Top., 10(1):3-29, 2005.

Walton, R. E. \& Vertucci, F. Anatomía Interna. En: Walton, R. E. \& Torabinejad, M. (Eds.). Endodoncia. Principios y Práctica Clínica. $2^{a}$ ed. México D. F., Mc Graw-Hill Interamericana, 1997. pp.177-91.

Dirección para Correspondencia:

Esther Vaillard Jiménez

Lázaro Cárdenas 61-A

Bello Horizonte

Cuautlancingo, Puebla

MÉXICO

Email: evaillard@gmail.com

Recibido : 07-01-2014

Aceptado: 26-03-2015 